K. MATSUZAKI

KODAI MATH. J.

$13(1990), 377-385$

\title{
CERTAIN ESTIMATES ON KLEINIAN GROUPS BY THE CORE OF THEIR QUOTIENT 3-MANIFOLD
}

Dedicated to Professor Tatsuo Fuji'i'e on his second birthday in the Chinese calendar

\author{
By Katsuhiko MatsuzaKi
}

\section{Introduction}

Kulkarni and Shalen [6] explained topologically Ahlfors' finiteness theorem and its two major supplements, the area-inequalities [2] and the finiteness of the cusps [16], considering the core of the quotient 3-manifold of finitely generated torsion-free Kleinian groups. As a consequence, they obtained the sharp inequalities on the number of cusps.

In this article we treat chiefly another result from the finiteness theorem. It is that every component subgroup of a finitely generated Kleinian group is also finitely generated. This assertion is qualitative. So we will estimate quantitatively the minimal number of generators of component subgroups. Our consequence $(\S 2)$ is ;

THEOREM. Let $G$ be a torsion-free Kleinian group with $r$ generators. Then for any component subgroup $H$ of $G$, the minimal number of generators of $H$ is not more than $2 r-1$.

Moreover we construct examples of Kleinian groups which attain the equalities in those estimates along the line from the finiteness theorem ( $\$ 3$ ).

Another part of this article is concerned with Ahlfors' measure zero problem (§4). Bonahon's theorem [3] shows us a sufficient condition for the limit set to be null on the 2-dimensional Lebesgue measure. With the aid of this result, we have;

THEOREM. Let $G$ be a torsion-free Kleinian group with $r$ generators. If \{the hyperbolic area of $\Omega(G) / G\} \geqq 4 \pi(r-2)$, then the limit set $\Lambda(G)$ has measure zero.

I want to thank the referee for his careful reading and valuable suggestions.

\section{$\S 1$. Definitions.}

In this paper, Kleinian groups are finitely generated and torsion-free discrete

Received November 2, 1989; revised April 12, 1990. 
subgroups of Möbius transformations of the second kind. Let $G$ be a Kleinian group. We denote the region of discontinuity by $\Omega(G)$ and the limit set by $\Lambda(G)$. Its action on the Riemann sphere can be naturally extended to the upper half space $H^{3}$. Its quotient bordered 3-manifold is denoted by $M(G)$; that is, $M(G)=H^{3} \cup(\Omega) / G$. Let $\Delta$ be a connected component of $\Omega(G)$. Then we call the stabilizer of $\Delta$ in $G$ the component subgroup for $\Delta$. Consider the Riemann surface $\Delta / \operatorname{stab}(\Delta)$. It is a component of $\partial M(G)$. Ahlfors' finiteness theorem asserts that $\partial M(G)$ consists of a finite number of components and each of them is analytically finite.

Although $M(G)$ may not be compact in general, by Scott's theorem, there is a compact submanifold of $M(G)$ which is homotopy equivalent to $M(G)$. We call such a compact submanifold a core of $M(G)$. Kulkarni and Shalen's work was dene by constructing a core which contains the compact part of $\partial M(G)$ under some assumptions. Their argument was refined by McCullough to the following form.

Proposition 1.1 ([14]). Let $Y$ be a 3-manifold with finitely generated fundamental group. Let $C$ be a compact submanifold of $\partial Y$. Then there is a core $X$ of $Y$ such that $X \cap \partial Y=C$.

A cusp in $M(G)$ is the projection of some region in $H^{3} \cup \Omega(G)$ associated with a parabolic fixed point (cf. [8, §2]). It is homeomorphic to (cylinder) $\times$ $(0,1)$ or (torus) $\times(0,1)$, according as the parabolic subgroup is isomorphic to $\boldsymbol{Z}$ or $\boldsymbol{Z}+\boldsymbol{Z}$. We call its border in $M(G)$ a cusp cylinder or cusp torus respectively.

By Proposition 1.1, we can take a core of $M(G)$ - cuspidal parts\}, which is also a core of $M(G)$ so that it may contain all the $\partial M(G)$ except for small neighborhoods of punctures on $\partial M(G)$, all the cusp cylinders except for open ends, and all the cusp tori. The core which has such a property is denoted by $N=N(G)$. For the detailed construction, see [12]. The cuspidal parts in $\partial N$ is denoted by $\partial_{p} N$. Therefore $\partial N-\partial_{p} N$ consists of topologically finite surfaces in $\partial M$ and "degenerate" surfaces.

Notations.

$r:=$ the rank of a Kleinian group, i. e. the minimal number of generators

$b:=\#\left\{\right.$ components of $\partial M$ or $\left.\partial N-\partial_{p} N\right\}$

$\chi:=(-1) \times\{$ Euler characteristic $\}$

$\beta_{\imath}:=$ the $i$-th Betti number

$m:=$ Lebesgue measure on $\boldsymbol{C}$.

\section{$\S 2$. The ranks of component subgroups}

We begin with starting a useful proposition for our estimates (cf. [4]).

Proposition 2.1. Let $G$ be a Kleinian group with $r(G)=r$. Then the fol- 
lowings are equivalent:

(a) $G$ is free.

(b) $\beta_{1}(M(G))=r$ and $\beta_{2}(M(G))=0$.

Proof. (a) $\rightarrow$ (b): Since $\pi_{1}(N(G))$ is free, we known $N(G)$ is a handlebody of genus $r$ [5, Ch. 5]. Then $\beta_{1}(M(G))=\beta_{1}(N(G))=r$ and $\beta_{2}(M(G))=\beta_{2}(N(G))$ $=0$. (b) $\rightarrow($ a) : Because $M(G)(N(G))$ is aspherical [8, p. 388], the homology groups of $G$ of coefficient $Z$ with trivial action are isomorphic to the ordinary homology groups of $M(G)$ (cf. [5, p. 75]). That is, $H_{i}(G, \boldsymbol{Z}) \cong H_{i}(M(G), \boldsymbol{Z})(i=1,2, \cdots)$. Since $M(G)$ is homotopy equivalent to a 2-dimensional $C W$-complex, $\beta_{2}(M(G))=0$ implies that $H_{2}(G, \boldsymbol{Z}) \cong H_{2}(M(G), \boldsymbol{Z})=0$. Here we use the following lemma [15, p. 179]. "Let $G$ be a group such that $H_{2}(G, Z)=0$. Let $\left\{x_{j}\right\}$ be a set of elements of $G$ whose images under the canonical epimorphism $\varphi: G \rightarrow H_{1}(G, Z)$ are linearly independant over $\boldsymbol{Z}$. Then $\left\{x_{j}\right\}$ is a basis of a free subgroup of $G$ ". Let $\left\{x_{j}\right\}$ $=\left\{x_{1}, \cdots, x_{r}\right\}$ be a minimal system of generators of $G$. To apply the lemma to our case, we have only to check that $\varphi\left(x_{1}\right), \cdots, \varphi\left(x_{r}\right)$ are linearly independent over $\boldsymbol{Z}$. It is easy: They generate $H_{1}(G, \boldsymbol{Z})$, for $\varphi$ is surjective. And rank $H_{1}(G, \boldsymbol{Z})=\beta_{1}(M(G))=r$ means that they are linearly independent.

Bers [2] proved that for a finitely generated Kleinian group $G$ which may contain elliptic elements, the hyperbolic area of $\Omega(G) / G$ is bounded by $4 \pi(r(G)-1)$. In the case without torsion, this theorem can be explained simply by using the core $N(G)$. Further, the condition which attain the equality is obtained. The result itself is originally due to Abikoff [1]:

Proposition 2.2 (Area-inequalities). Let $G$ be a Kleinian group. Then, $\chi(\partial M(G)) \leqq \chi(\partial N(G)) \leqq 2(r(G)-1)$. The first equality holds if and only if $G$ is geometrically finite. The second equality holds if and only if $G$ is free.

Proof. By Poincaré's duality theorem, we know $\chi(\partial N(G))=2 \chi(N(G))$. Let $\beta_{2}=\beta_{i}(N(G))=\beta_{i}(M(G))$. Since $\beta_{0}=1, \beta_{3}=0, \beta_{1} \leqq r$ and $\beta_{2} \geqq 0$, it follows that $\chi(\partial M) \leqq \chi(\partial N)=2\left(\beta_{1}-\beta_{2}-1\right) \leqq 2(r-1)$. By Marden's characterization of geometric finiteness [8, Prop. 4.2], $\chi(\partial M(G))=\chi(\partial N(G))$ if and only if $G$ is geometrically finite. $2\left(\beta_{1}-\beta_{2}-1\right)=2(r-1)$ if and only if $\beta_{1}=r$ and $\beta_{2}=0$. By Proposition 2.1, it is that $G$ is free.

Now we prove our main theorem mentioned in the introduction.

THEOREM 2.3. Let $G$ be a non-elementary Kleinian group with $r(G)=r$. Then for any component subgroup $H$ of $G, r(H) \leqq 2 r-1$. The equality holds if and only if (1) $G$ is geomertically finite and $\partial M(G)$ is connected, (2) $G$ is free, and (3) $\partial M(G)$ is incompressible in $M(G)$. Further, there are examples for every $r \geqq 2$, which attain the equality. 
Remark. (3) is equivalent to the fact that all the components of $\Omega(G)$ are simply connected. $\partial M(G)$ is connected if and only if all the components of $\Omega(G)$ are conjugate to one another by $G$.

Proof. Let $H=\operatorname{stab}(\Delta)$ and $S=\Delta / H$. We consider two cases; $S$ is not closed, or $S$ is closed. (I) If $S$ is not closed, then $r(H) \leqq r\left(\pi_{1}(S)\right)=\chi(S)+1$. Since $\chi(S) \leqq \chi(\partial N)$ and $\chi(\partial N) \leqq 2(r-1)$ (Proposition 2.2), we see $r(H) \leqq 2 r-1$. (II) If $S$ is closed, then $r(H) \leqq r\left(\pi_{1}(S)\right)=\chi(S)+2$. Here we may assume that $G$ is not free, for if $G$ is free and $\partial M(G)$ has a closed surface, $G$ must be a Schottky group, hence $G=H$ and $r(H)=r$. By Proposition 2.1, it implies either $\beta_{1}<r$ or $\beta_{2}>0$ that $G$ is not free, thus $\chi(\partial N) \leqq 2(r-2)$. In this case, $r(H) \leqq \chi(S)+2 \leqq \chi(\partial N)$ $+2 \leqq 2 r-2<2 r-1$. Note that in (II), the equality $r(H)=2 r-1$ cannot occur.

We investigate the condition for the equality. From Proposition 2.2, (1) is equivalent to the fact that $\chi(S)=\chi(\partial N)$, and (2) is $\chi(\partial N)=2(r-1)$. (3) is a sufficient condition for $r\left(\pi_{1}(S)\right)=r(H)$, Thus the equality holds if (1), (2) and (3) are satisfied. Conversely if the equality holds, then by the above notice, $S$ is not closed, i.e. only (I) is in our attention. For the equality, it is necessary that $r(H)=r\left(\pi_{1}(S)\right), \chi(S)=\chi(\partial N)$ and $\chi(\partial N)=2(r-1)$ are satisfied. From the last two equalities, (1) and (2) are derived. We have only to show (3). Assume that $S$ is compressible. Then there are some relations in $\pi_{1}(S)$ by which $H$ is the quotient group of $\pi_{1}(S)$. Here $H$ is free, because $G$ is free by

(2) which has been already obtained. So $H$ has less rank than $\pi_{1}(S)$ [7, Prop. 2.7]. From this argument, we know $S$ must be incompressible for the equality.

The examples of sharpness for $r \geqq 2$ will be discussed in the following section (Example 2).

\section{$\S 3$. Other inequalities and examples of sharpness}

In this section we exhibit the examples of Kleinian groups which attain the equality in Theorem 2.3. Incidentally we also show that the area-inequalities (§2.) and the following two estimates are sharp. They are corresponding respectively to Theorems 2,3 and 4 of Abikoff [1], which are shown under some assumption. (See also Marden $[8, \S 7]$.) The assumption can be removed by McCullough's work. For the convenience of the readers, we now sketch their proofs here.

Proposition 3.1. Let $G$ be a non-elementary Kleinian group. Then, $b(\partial M(G))$ $\leqq 2(r(G)-1)$. The equality holds if and only if $G$ is geometrically finite, free and all the components of $\partial M(G)$ are thrice punctured spheres or once punctured tori.

Proof. For each component $S$ of $\partial M(G), \chi(S) \geqq 1$. It follows that $b(\partial M(G))$ $\leqq \chi(\partial M(G))$. The equality holds if and only if $\chi(S)=1$ for any $S$. Combining with Proposition 2.2, we have the assertion. 
Proposition 3.2 (Finiteness of Cusps). Let $G$ be a non-elementary Kleinian group. Then, \# \{conjugacy classes of parabolic fixed points $\leqslant 3(r(G)-1)$. (We count the fixed point of a parabolic abelian subgroup of rank 2 twice.) The equality holds if and only if $G$ is geometrically finite, free, and all the components of $\partial M(G)$ are thrice punctured spheres.

Proof. Let $\left\{S_{j}\right\}(j=1,2, \cdots, b)$ be the components of $\partial N(G)-\partial_{p} N(G)$. Let $g$, and $n$, be the numbers of handles and holes of $S_{j}$ respectively. Then we have $2\left(\beta_{1}-\beta_{2}-1\right)=\chi(\partial N)=\sum_{j=1}^{b} \chi\left(S_{j}\right)=\Sigma\left(2 g_{j}-2+n_{j}\right)$, where $\beta_{i}=\beta_{i}(N(G))$. Therefore $\#$ cusp cylinders $\}=(1 / 2) \Sigma n_{j}=\beta_{1}-\beta_{2}-1-\Sigma\left(g_{j}-1\right)$. Here $-\Sigma\left(g_{j}-1\right) \leqq b \leqq$ $2\left(\beta_{1}-\beta_{2}-1\right)$ by the same reason as Proposition 3.1. So \#\{cusp cylinders $\} \leqq$ $3\left(\beta_{1}-\beta_{2}-1\right)$. The equality is attained if and only if every $S$, is a sphere with three holes.

On the other hand \#\{cusp tori $\} \leqq \beta_{2}$, because they generate linearly independent elements in $H_{2}(N, \boldsymbol{Z})$. Hence, \#\{conjugacy classes of parabolic fixed points $\}=\#\{$ cusp cylinders $\}+2 \#\{$ cusp tori $\} \leqq 3\left(\beta_{1}-\beta_{2}-1\right)+2 \beta_{2}=3\left(\beta_{1}-1\right)-\beta_{2} \leqq$ $3(r-1)$.

It is now clear that it attains the equality if the conditions stated in this proposition are satisfied. For the converse, we have only to remark that a sphere with three holes cannot be a degenerate surface. It is because a Fuchsian group of signature $(0,3)$ has rigidity $[12, \S 5]$. If every $S$, is such a surface, then $G$ is geometrically finite.

All our examples are on boundaries of Schottky spaces. The theorem of Maskit on parabolic elements [11] guarantees that such groups are obtained by squeezing some simple disjoint primitive loops on the closed Riemann surfaces which bound the handlebodies.

Example 1. For every $r \geqq 2$, we construct a geometrically finite free Kleinian group $G$ with $r(G)=r$ such that all the components of $\partial M(G)$ are thrice punctured spheres. This is a sharp example for Propositions 2.2, 3.1 and 3.2.

Take a Fuchsian group of signature $(0, r+1)$. If $r=2$, it is the example. So we assume $r \geqq 3$. On the boundary of its Teichmüller space, there is a terminal regular $b$-group which has $r-2$ accidental parabolic transformations (A.P.T.) [9]. Then on the quotient surface of its invariant component, we can choose $r-2$ non-trivial, non-mutually-homotopic disjoint simple primitive loops, which correspond to loxodromic elements (see Fig. 1). Then by Maskit's theorem, there is a geometrically finite Kleinian group on the boundary of the deformation space, such that all those loops become representing parabolic elements. It has $(r+1)+(r-2)+(r-2)=3 r-3$ cusps, and all the components are thrice punctured spheres. 


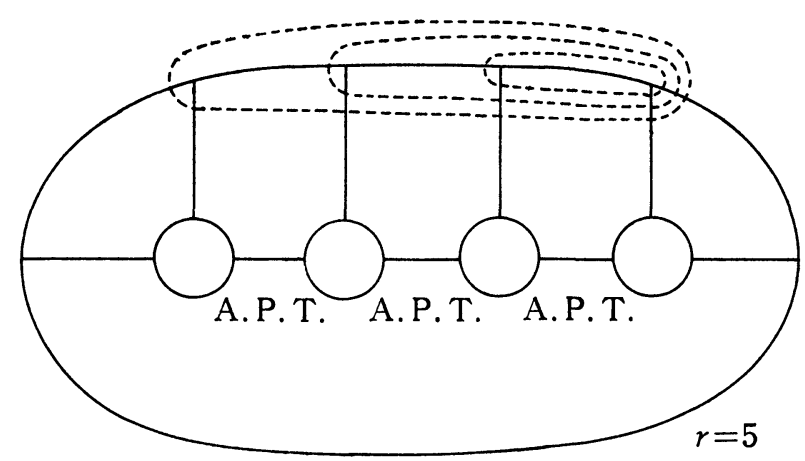

Fig. 1.

Example 2. First we consider the case $r=2$. Take two simple primitive loops on the border of a handlebody $N$ of genus 2 as in Fig. 2. When we denote two standard generators of $\pi_{1}(N)$ by $f$ and $g$, those two loops correspond to $f$ and $f g^{2}$. We can easily see that they don't divide $\partial N$. Moreover $\partial N-\{$ two loops $\}$ is incompressible in $N$. In fact, if not, there is a properly embedded disk in $N$ - two loops $\}$ which induces a non-trivial free product decomposition $\pi_{1}(N) \cong G=\langle p\rangle *\langle q\rangle$, such that all the parabolic elements of $G$ are in a conjugate of $\langle p\rangle$ or $\langle q\rangle$. This means that we can choose some conjugates of $f$ and $f g^{2}$ as generators of $G$. But it is impossible, because every word constructed by the generators must have an even integer as the sum of powers of $g$, so they cannot generate $g$ itself. For $r>2$, add appropriate $r-2$ cusp cylinders as in Fig. 2, so that $\partial N-\{$ loops $\}$ may be connected and incompressible. Then by Maskit's theorem, we obtain the Kleinian groups which are examples of sharpness in Theorem 2.3 for $r \geqq 2$.

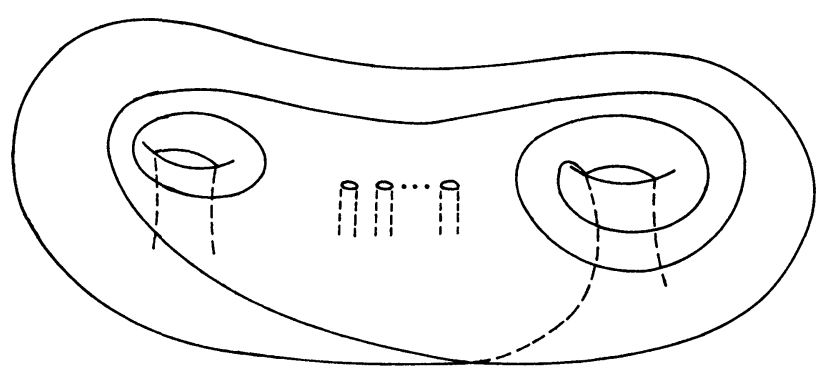

Fig. 2. 


\section{§4. The measure zero problem}

The remainder of this paper is concerned with Ahlfors' conjecture; that is, finitely generated Kleinian groups have the measure zero limit set.

Proposition 4.1. Let $G$ be a Kleinian group which is constructed from Kleinian groups satisfying the condition (*) by Klein's combination theorem. Then $m(\Lambda(G))=0$. The condition $(*)$ is as follows:

(*) For every non-trivial free product decomposition $G=A * B$, there is a parabolic element of $G$ which is not in a conjugate of $A$ or $B$.

Proof. This is a consequence from Bonahon's theorem [3] and the combination theorem [10].

LEMMA 4.2. Let $G$ be a free Kleinian group. Let $S$ be a component of $\partial N(G)-\partial_{p} N(G)$ which is compressible in $N(G)$. Then $\chi(S) \geqq 3$, or $G$ is geometrically finite.

Proof. Since $S$ is compressible, there is a properly embedded disk $D$ in $N$ which induces a non-trivial free product decomposition of $G$ with $\partial D \subset S$. Let $S-\partial D$ be $S_{1}$ and $S_{2}$ if $\partial D$ divides $S$, or only $S_{0}$ if $\partial D$ does not divide $S$. In the former case, we denote $S_{i} \cup \bar{D}$ by $\hat{S}_{\imath}(i=1,2)$. In the latter case, $S_{0}$ has topologically two boundary components corresponding to $\partial D$. $S_{0}$ to which two copies of $\bar{D}$ are attached is regarded as $\hat{S}_{0}$. We have $\chi(S)=\chi\left(\hat{S}_{1}\right)+\chi\left(\hat{S}_{2}\right)+2$ if $\partial D$ divides $S$, or $\chi(S)=\chi\left(\hat{S}_{0}\right)+2$ if $\partial D$ does not divide $S$.

For $i=0,1,2, \chi\left(\hat{S}_{\imath}\right)=-2$ does not occur since $N$ is irreducible, and neither does $\chi\left(\hat{S}_{2}\right)=-1$ since a loop round the hole is not homotopically trivial. Hence $\chi\left(\hat{S}_{\imath}\right) \geqq 0$. Assume that $\chi(S) \leqq 2$. It follows that $\chi\left(\hat{S}_{\imath}\right)=0$ for $i=0,1,2$. Then $\hat{S}_{i}$ is either a torus or a sphere with two holes. In the latter case, a loop round a hole is freely homotopic in $N$ to a loop round the other hole. Therefore they correspond to the same conjugacy class of primitive parabolic elements. It means that these two holes are connected with a cusp cylinder.

If necessary, by jointing such a cusp cylinder to $\hat{S}_{\imath}$, we may assume that $\hat{S}_{\imath}$ is a torus. Since $G$ is free, it bounds a solid torus. Hence $N$ is a handlebody of genus 2 , and $S$ is the only component of $\partial N-\partial_{p} N$. Further $S \subset \partial M(G)$, for $G$ has the region of discontinuity. Therefore $G$ is geometrically finite by Marden's characterization.

THEOREM 4.3. Let $G$ be a Kleinian group with $r(G)=r$. If $\chi(\partial M(G)) \geqq$ $2(r-2)$, then $m(\Lambda(G))=0$.

Proof. Consider components of $\partial N-\partial_{p} N$. Bonahon's condition (*) is equivalent to the fact that all the components of $\partial N-\partial_{p} N$ are incompressible in $N$ 
(see $[3$, p. 72$]$ ). Then we know $G$ does not satisfy the assumption of Proposition 4.1 if and only if there exists a compressible component of $\partial N-\partial_{p} N$ which is not contained in $\partial M$.

In the case $G$ is free, by Lemma 4.2 , such a compressible component $S$ has $\chi(S) \geqq 3$. Then $\chi(\partial M(G)) \leqq \chi(\partial N)-\chi(S) \leqq 2(r-1)-3<2(r-2)$. In the case $G$ is not free, by Proposition $2.1, \beta_{1}<r$ or $\beta_{2}>0$. It follows that $\chi(\partial N) \leqq 2(r-2)$. It is clear that $\chi(S) \geqq 1$. Hence $\chi(\partial M(G)) \leqq \chi(\partial N)-\chi(S) \leqq 2(r-2)-1<2(r-2)$. Therefore if $\chi(\partial M(G)) \geqq 2(r-2)$, degenerate compressible surfaces can not exist.

CoRollaRy 4.4 ([13]). If $r(G)=2$, then $m(\Lambda(G))=0$.

Corollary 4.4 means that if $r(G)=2$, then $\partial N(G)-\partial_{p} N(G)$ has no degenerate compressible surface. But if $r(G)=3$, it may consist of two surfaces $S_{1}$ and $S_{2}$, $S_{1}$ is not degenerate with $\chi\left(S_{1}\right)=1$ and $S_{2}$ is degenerate with $\chi\left(S_{2}\right)=3$. The following theorem asserts that such a group actually exists.

THEOREM 4.5. There is an example of the three generator Kleinian group $G$ which is geometrically infinite, does not satisfy the condition (*), and whose $\partial M(G)$ consists of one incompressible surface.

Proof. Let $H$ be a Fuchsian group of the first kind with two free generators, acting on the unit disk $D$. Let $f_{0}$ be a loxodromic element such that the isometric circles of $f_{0}$ and $f_{0}^{-1}$ are contained in $D$ and the distance of two fixed points of $f_{0}$ is small enough. Then by the combination theorem [10], $G_{0}=\left\langle H, f_{0}\right\rangle$ are geometrically finite Kleinian group and $G_{0}=H *\left\langle f_{0}\right\rangle$. Let $f_{t}=$ $g_{t} f_{0} g_{t}{ }^{-1}$, where $\left\{g_{t}\right\}(t \geqq 0)$ is a continuous path starting at $i d$. in $P S L(2, \boldsymbol{C})$. We choose $\left\{g_{t}\right\}$ so that the Euclidian distance between the fixed points of $f_{t}$ may be larger to infinity as $t$ increases, and all the parabolic elements of $G_{t}=$ $\left\langle H, f_{t}\right\rangle$ are conjugate to elements in $H$. This can be done because only a countable number of points in $P S L(2, C)$ violate our second requirement.

Then $G_{t}$ is a quasiconformal $(Q C)$ deformation of $G_{0}$ when $t$ is small, for $G_{0}$ is $Q C$ stable [8, Prop. 9.1]. But there exists

$$
T=\sup \left\{t \mid \text { for all } s \in[0, t), G_{s} \text { is q. c. deformation of } G_{0}\right\}
$$

before a fixed point of $f_{t}$ hits at $\partial D$. Let $f_{T}$ be $f$ and $G_{T}$ be $G$.

Since $G_{t}(t<T)$ has $\Delta=\{|z|>1\} \cup\{\infty\}$ as its component, it is easy to see that $\Delta$ is a component of the region of discontinuity of $G$, particularly $G$ is Kleinian. Moreover since $G_{t}$ is $H *\left\langle f_{t}\right\rangle$ when $t<T$ and $G_{t}$ converges algebraically to $G$, we know $G=H *\langle f\rangle$. Therefore $G$ does not satisfy the condition (*), for any parabolic element of $G$ is in a conjugate of $H$. Further $G$ is geometrically infinite, for if $G$ were geometrically finite, then $G$ would be $Q C$ stable: it contradicts our definition of $T . S=\Delta / H$ is an incompressible components of $\partial M(G)$ with $\chi(S)=1$. By Lemma 4.2, we can see that $\partial M(G)$ has no other components than $S$, because $\chi(\partial N(G))=4$. 


\section{REFERENCES}

[1] Aвikoff, W., The Euler characteristic and inequalities for Kleinian groups, Proc. Amer. Math. Soc. 97 (1986), 593-601.

[2] BERs, L., Inequalities for finitely generated Kleinian groups, J. Analyse Math. 18 (1967), 23-41.

[3] Bonahon, F., Bouts des variété hyperbolique de dimension 3, Ann. of Math. 124 (1986), 71-158.

[4] Gusevskil, N.A., Topological characterization of Schottky groups, Siberian Math. J. 20 (1979), 454-457.

[5] Hempel, J., 3-Manifolds, Ann. of Math. Studies 86, 1976.

[6] Kulkarni, R.S. and Shalen, P.B., On Ahlfors' finiteness theorem, Advances in Math. 76 (1989), 155-169.

[7] Lyndon, R.C. AND Schupp, P.E., Combinatorial Group Theory, Springer-Verlag, 1977.

[8] MARDEN, A., The geometry of finitely generated Kleinian groups, Ann. of Math. 99 (1974), 383-462.

[9] Maskit, B., On boundaries of Teichmüller spaces and on kleinian groups: II, Ann. of Math. 91 (1970), 607-639.

[10] Maskit, B., On Klein's combination theorem III, Ann. of Math. Studies 66, (1971). 297-316.

[11] Maskit, B., Parabolic elements in Kleinian groups, Ann. of Math. 117 (1983), 659-668.

[12] Maskit, B. AND Swarup, G., Two parabolic generator Kleinian groups, Israel J. Math. 64 (1988), 257-266.

[13] MatsuzaKi, K., A characterization of extended Schottky type groups with a remark to Ahlfors' conjecture, to appear in J. Math. Kyoto Univ 31 (1991).

[14] McCullough, D., Compact submanifolds of 3-manifolds with boundary, Quart. J. Math. 37 (1986), 299-307.

[15] Stallings, J., Homology and central series of groups,. J. Algebra 2 (1965), 170-181.

[16] Sullivan, D., A finiteness theorem for cusps, Acta Math. 147 (1981), 289-299.

Department of Mathematics, Faculty of Science,

Kyoto University, Kyoto 606, JAPAN

CURRENT AdDRESS

Department of Mathematics, Tokyo Institute of Technology

Oh-okayama, Meguro, Tokyo 152, Japan 